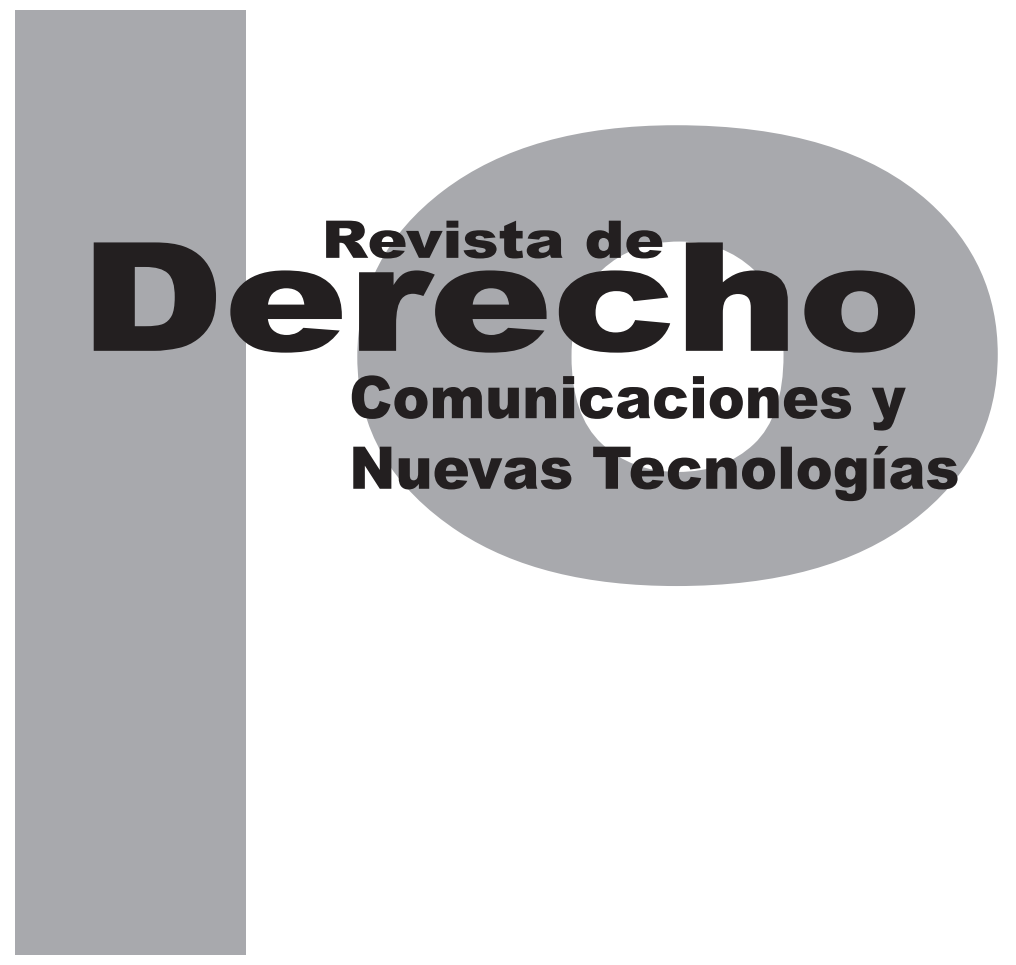

\title{
LA IMPORTANCIA DE FOMENTAR LA COOPERACIÓN INTERNACIONAL EN LA UTILIZACIÓN Y EXPLORACIÓN DEL ESPACIO ULTRATERRESTRE
}

\author{
VALentina VARELA Martínez
}

Universidad de los Andes

Facultad de Derecho

Revista de Derecho, Comunicaciones y Nuevas Tecnologías

No. 10, Julio - Diciembre de 2013. ISSN 1909-7786 


\title{
La importancia de fomentar la cooperación internacional en la utilización y exploración del espacio ultraterrestre*
}

\author{
Valentina Varela Martínez ${ }^{* *}$
}

\section{RESUMEN}

El trabajo que se presenta a continuación tiene como fin exaltar la importancia que tienen la cooperación, la confianza y el consenso en las relaciones que naturalmente surgen entre los Estados en el marco de las actividades que se adelantan en el Espacio Ultraterrestre. Teniendo presente que la dinámica del Derecho Espacial se vive más allá de los $100 \mathrm{~km}$ sobre el nivel del mar y que por lo tanto, el grado de inspección, vigilancia y actuación con el que cuentan los diferentes Estados en ese lugar es mucho menor del que tienen sobre los sucesos que ocurren en la tierra, surge la necesidad de conducir la actividad espacial bajo la premisa de que, por
The following work has the purpose of emphasizing the importance that cooperation, trust and consensus have within relationships that occur naturally between the States on the margin of the activities that take place in the Ultra terrestrial Space. Taking into consideration that the dynamic of the spatial law takes place further than $100 \mathrm{~km}$ over the sea level, and that because of that, the degree of inspection, vigilance and action which the various States count on within this location is far lower than the one they have over the events that take place on lower ground, the need to conduct spatial activity under the premise that simply by being part of the

Cómo citar este artículo: Varela Martínez, V. (Diciembre, 2013). La importancia de fomentar la cooperación internacional en la utilización y exploración del espacio ultraterrestre. Revista de Derecho, Comunicaciones y Nuevas Tecnologías, 10.

** Estudiante de Derecho, próxima a graduarse (Diciembre de 2013) de la Universidad Javeriana Cali. Asistente jurídica de la Clínica Jurídica de Litigio Estratégico -GLE- de la Pontificia Universidad Javeriana Cali (Julio, 2012 - Julio, 2013). Miembro del Consejo Editorial del periódico de la carrera de Derecho "Panorama Javeriano" de la misma universidad (Junio, 2011 - Julio, 2013). vvarela@javerianacali.edu.co 
el sólo hecho de ser parte de Naciones Unidas y haber consensuado los diferentes Convenios, Declaraciones y Tratados sobre esta materia, los Estados quedan obligados y sujetos a ellos. Y más allá, dan su palabra de orientar sus políticas internas y cada una de sus actuaciones de acuerdo al principio de cooperación y asistencia mutua.

Palabras clave: Cooperación, confianza, consenso, relaciones internacionales, espacio.
United Nations and having agreed to the various treaties and declarations on this matter, the States are therefore under obligation and ruled by them. And furthermore they give their word and agree to guide their internal policies and each of their actions according to the cooperation and mutual assistance principle.

KEYwORDS: Cooperation, trust, consensus, international relations, space. 


\section{SUMARIO}

Introducción - I. LA COOPERACIÓN INTERNACIONAL EN EL MARCO DE LAS RELACIONES INTERNACIONALES - II. LA COOPERACIÓN INTERNACIONAL EN LA EXPLORACIÓN Y UTILIZACIÓN DEL ESPACIO ULTRATERRESTRE - A. ¿Por qué debe haber cooperación en el espacio ultraterrestre? -1. Evolución de la cooperación internacional en el espacio ultraterrestre -B. La cooperación como estrategia para fomentar la confianza entre los Estados - 1. Al referirnos a la confianza, ¿de qué hablamos? - 2. La confianza como elemento esencial en la exploración y utilización del espacio ultraterrestre - 3 . El consenso como generador de confianza - 4. ¿Cómo lograr esa confianza? - 4.1. Un constante intercambio de información - 4.2. Un sistema de notificaciones previas -4.3. Una evaluación y modificación del actual régimen jurídico del espacio ultraterrestre - C. La cooperación internacional como norma de lus Cogens - III. CONCLUSIONES -Bibliografía. 
Introducción

Desde que el término existe, la cooperación internacional siempre ha hecho referencia al trabajo en comunidad y a la unión de esfuerzos y recursos entre los diferentes países del mundo que deciden colaborar en la ejecución de proyectos y nuevas estrategias que, después de un tiempo de dedicación, les representan beneficios, sobre todo a nivel de desarrollo. Tradicionalmente, los campos cubiertos por la cooperación internacional han sido aquellos relacionados con la salud, la educación, las condiciones ambientales y las desigualdades que se presentan en el ámbito social y económico.

Sin embargo, como producto de los múltiples adelantos científicos y tecnológicos, y con ellos, las dificultades en nuevas áreas del derecho, ha sido necesario que el concepto de cooperación internacional también sea utilizado para regular todos los eventos que suceden más allá de los $100 \mathrm{~km}$ sobre el nivel del mar, o en otras palabras, aquellos que tienen lugar en el espacio ultraterrestre.

\section{LA COOPERACIÓN INTERNACIONAL EN EL MARCO DE LAS RELACIONES INTERNACIONALES}

Para nadie es un secreto que la globalización ha sido uno de los temas más cotizados en varios de los debates internacionales de los últimos tiempos. Aun cuando no existe una "definición exacta y ampliamente aceptada" (Banco Mundial, 2000, párr. 2), este fenómeno ha sido in- terpretado por diversas organizaciones internacionales. El Banco Mundial, por ejemplo, lo sitúa en un ámbito puramente económico, al sostener que: "en los últimos años una parte de la actividad económica del mundo, que aumenta en forma vertiginosa, parece estar teniendo lugar entre personas que viven en países diferentes" (Banco Mundial, 2000, párr. 2). Por otro lado, la Comisión Económica para América Latina y el Caribe (CEPAL) no sólo ha reconocido el vínculo que indudablemente tiene la globalización con la economía, sino que también la ha asociado con la "creciente gravitación de procesos económicos, sociales y culturales de carácter mundial (CEPAL, 2002, párr. 1).

Así como no existe un acuerdo en relación a su interpretación, también se presenta una disputa respecto de los efectos de la globalización. En este sentido, hay quienes sostienen que son más las ventajas, mientras otros hacen énfasis en sus efectos negativos. Quienes se inclinan por los riesgos y las repercusiones desfavorables resaltan el incremento de la desigualdad entre países y el aumento del desempleo (Mayoral, (s.f.)). Sumado a eso, sobresale la inestabilidad que a nivel comercial y financiero termina excluyendo a los países que no están preparados para asumir las demandas del mundo contemporáneo.

Es en medio de este amplio panorama que surge la necesidad de lograr interacciones, de todo tipo, entre los diferentes países del mundo que estén dirigidas a obtener un mayor crecimiento y progreso social (Espinosa Acuña y Vaca Gonzalez, 2012, Comercio Exterior, Cooperación In- 
ternacional y Progreso Técnico desde la Teoría del Desarrollo Económico de la CEPAL y de Raúl Prebisch, p. 31) dando origen a lo que hoy se conoce, en el marco de las relaciones internacionales, como la cooperación Internacional.

Por medio de estas interacciones y del ejercicio de la ayuda mutua, los distintos países luchan por reducir la desigualdad social y económica y mejorar la calidad de vida de todos sus habitantes, sobre todo la de aquellos que pertenecen a los sectores más vulnerables y para ello buscan "lograr mayores niveles de inversión en investigación y tecnología capaces de influir en la generación de industria, producción y desarrollo sostenible"(Espinosa Acuña y Vaca Gonzalez, 2012, p. 31).

Al respecto, los Estados que han entrado a conformar esta dinámica, han decidido incorporar en su legislación interna una serie de principios y normas orientadas a fortalecer la asistencia mutua, la complementariedad y el respeto por "los bienes públicos globales" como es el caso de la paz, la seguridad, la justicia, la democracia y la sostenibilidad ambiental"(Espinosa Acuña y Vaca Gonzalez, 2012, p. 32).

\section{LA COOPERACIÓN INTERNACIONAL EN LA EXPLORACIÓN Y UTILIZACIÓN DEL ESPACIO ULTRATERRESTRE}

\section{A. ¿Por qué debe haber cooperación en el espacio ultraterrestre?}

Son muchas las razones que podemos citar para afirmar que en todo el ejercicio de la actividad que se desarrolla en el marco del espacio ultraterrestre debe existir un clima de cooperación. Sin embargo, es preciso señalar dos de ellas:

La primera tiene relación directa con la evolución que ha tenido la actividad en el espacio ultraterrestre, que básicamente ha trascendido de "la etapa exploratoria a la del uso práctico del mismo" (Consejo de Estudios Internacionales Avanzados, 1981, Mesa redonda sobre solución de controversias en derecho espacial, p. 9). Al principio, los Estados centraron todos sus esfuerzos en explorar ese nuevo universo al que habían podido acceder. En ese momento, la preocupación solo consistía en establecer un orden y una manera de guiar las relaciones, de allí “que el Tratado del Espacio pudo en gran medida adoptarse y obtener una amplia ratificación" (Consejo de Estudios Internacionales Avanzados, 1981, p. 10). No obstante, hoy en día, superada la fase exploratoria y estando vigente la etapa práctica de los adelantos a nivel de la ciencia y la tecnología, las preocupaciones son otras. Debido a la participación de nuevos Estados y a la constante competencia de las viejas potencias, actualmente los esfuerzos se concentran en los diferentes intereses políticos 
y económicos que tienen cada uno de los Estados y que son los que impiden, precisamente, los nuevos consensos. (Consejo de Estudios Internacionales Avanzados, 1981, p. 10).

La segunda razón, hace referencia a que en el espacio ultraterrestre existen aspectos como la órbita de los satélites geoestacionarios que se caracterizan por ser recursos únicos y limitados. Por esta razón, su regulación debe ser distinta a la del resto de los elementos que constituyen el espacio ultraterrestre, garantizando específicamente un acceso equitativo a la órbita (Rodríguez Medina, (s.f.), Nuestro Derecho al Espacio. La órbita geoestacionaria: ¿una frustrada regulación?, p. 70), su uso pacífico y la no apropiación del recurso. En este sentido, es evidente que para lograr dichos fines, más que consenso, debe mediar una cooperación entre los Estados.

\section{Evolución de la cooperación internacio- nal en el espacio ultraterrestre}

El camino hacia la cooperación internacional en la actividad espacial ha sido largo y ha contado con la participación de muchos organismos internacionales que han contribuido a solidificarlo.

Los primeros pasos de este proceso se dieron en 1950, al establecerse la Federación Internacional de Astronáutica (Lachs, 1977, p. 42) que fue fundada con el objetivo de fomentar el desarrollo de la astronáutica con fines pacíficos y promover la cooperación internacional en esta materia, ayudar a difundir información relacionada con las dinámicas que se adelantan en el espacio ultraterrestre e impulsar la investigación en asuntos de astronáutica.

Para esa misma época, sucedió en el mundo algo que para muchos significó un verdadero logro de la tecnología: el lanzamiento de los primeros satélites y vehículos espaciales. Este tipo de adelantos científicos-tecnológicos, empezaron a evidenciar la urgencia de contar con una coordinación en la investigación espacial.

Ante esta necesidad y para poder ofrecer a la comunidad científica mundial "los medios necesarios para explotar las posibilidades de los satélites y las sondas espaciales de todo tipo para fines científicos e intercambiar los resultados sobre la base de la cooperación" (Lachs, 1977, p. 43), en 1958, el Consejo Internacional para la Ciencia creó el Comité de Investigación Espacial (COSPAR). Como tareas importantes, al comité le fueron asignadas: la promoción de la investigación científica del Espacio Ultraterrestre y la promoción de un foro abierto a todos los científicos del espacio para la discusión de circunstancias que afectaran y pudieran Ilegar a afectar en un futuro la actividad en el Espacio Ultraterrestre.

Asimismo, en 1959 la Asamblea General de Naciones Unidas creó la Comisión sobre la Utilización del Espacio Ultraterrestre con fines pacíficos y le asignó las siguientes funciones:

- Revisar en forma adecuada el área de la cooperación internacional y estudiar los medios prácticos y factibles para llevar a cabo los programas para el uso pacífico del Espacio Ultraterrestre. 
- Estudiar la naturaleza de los problemas legales que llegaran a surgir en la exploración y utilización del Espacio Ultraterrestre. (Asamblea General de las Naciones Unidas, 1959, Cooperación internacional en la utilización del espacio ultraterrestre con fines pacíficos, Art. 1).

Para poder cumplir con sus responsabilidades, la Comisión creó dos subcomisiones. La primera, la Subcomisión de Asuntos Científicos que se encarga, básicamente, del intercambio y difusión de información, de adelantar programas internacionales sobre investigación espacial, de fomentar la preparación de científicos en el campo del espacio y de calificar el trabajo desarrollado por las organizaciones especializadas que dedican su tiempo y sus recursos a la investigación del espacio (Lachs, 1977, p. 54). La segunda, la Subcomisión de Asuntos Jurídicos, se ocupa de diseñar los medios necesarios para garantizar la utilización equitativa de la órbita geoestacionaria y de solucionar los problemas que, a nivel legal, puedan surgir entre los Estados en el desarrollo de la actividad en el Espacio Ultraterrestre.

Los resultados de todas las actuaciones emprendidas por el Comisión se reflejaron en dos documentos importantes: la Declaración de los Principios Legales que rigen las Actividades de los Estados en la Exploración y uso del Espacio Ultraterrestre, adoptada por la Asamblea General de Naciones Unidas el 13 de diciembre de 1963 y el Tratado sobre los Principios que deben regir las Actividades de los Estados en la Exploración del Espacio Ultraterrestre, incluida la Luna y otros Cuerpos Celestes, aprobado el 19 de diciembre de 1966.
La declaración de 1963 surgió por la necesidad de diseñar un conjunto de principios capaz de regular la actividad espacial y tendiente a garantizar un régimen jurídico bajo la filosofía de Naciones Unidas. Si bien la declaración representaba un gran avance, desafortunadamente no tenía fuerza vinculante, lo que hacía de su adopción y respeto una elección de cada Estado.

Por esta razón, se hizo necesario que los principios, ya propuestos en la declaración, fueran nuevamente adoptados, pero esta vez en un tratado que pudiera obligar a cada uno de los Estados parte (Kopal, 2009, p. 2). Fue así como se creó el tratado de 1967 conocido por muchos como el Tratado del Espacio, donde quedaron consagrados los siguientes principios:

- Actividad en beneficio de toda la humanidad;

- Igualdad para todos los Estados;

- No soberanía;

- Uso pacífico;

- Cooperación internacional;

- Responsabilidad por daños causados.

De manera simultánea, Naciones Unidas elaboró otros instrumentos jurídicos más específicos para la regulación del espacio ultraterrestre:

- Acuerdo sobre el salvamento y la devolución de astronautas y la restitución de objetos lanzados al Espacio Ultraterrestre, aprobado el 19 de diciembre de 1967; 
- Convenio sobre responsabilidad internacional por daños causados por objetos espaciales, aprobado el 29 de noviembre de 1971;

- Convenio sobre el registro de objetos lanzados al Espacio Ultraterrestre, aprobado el 9 de diciembre de 1974;

- Acuerdo que debe regir las actividades de los estados en la luna y otros cuerpos celestes, aprobado en 1979.

Por último, con el ánimo de recopilar las disposiciones normativas en materia de cooperación de cada uno de los tratados que constituyen las fuentes de derecho del Espacio Ultraterrestre, Naciones Unidas aprobó, el 4 de febrero de 1997, la Declaración sobre la cooperación internacional en la exploración y utilización del Espacio Ultraterrestre en beneficio e interés de todos los Estados, teniendo especialmente en cuenta las necesidades de los países en desarrollo. De esta declaración, sobresalen los siguientes aspectos:

- La cooperación internacional debe siempre ponerse en práctica de conformidad con las disposiciones de derecho internacional, más específicamente con aquellas contenidas en la Carta de Naciones Unidas y el Tratado del Espacio de 1967;

- La cooperación debe realizarse en beneficio y teniendo en cuenta los intereses de cada uno de los Estados, sin necesidad de considerar su grado de desarrollo social, económico, científico y técnico (Naciones Unidas, 1997). No obstante, al tratarse de una cooperación en beneficio de toda la humanidad, se le debe dar especial atención a las necesidades de los países en vía de desarrollo.
- En materia de cooperación y asistencia mutua, son los mismos Estados los que determinan su grado de participación y las modalidades a través de las cuales quieren llevar a cabo dicha cooperación. Los países escogerán aquellas que "consideren más eficaces y adecuadas incluidas, entre otras, la cooperación gubernamental y no gubernamental; comercial y no comercial; mundial, multilateral, regional o bilateral y la cooperación internacional entre países de distintos niveles de desarrollo" (Asamblea General de las Naciones Unidas, 1997, Art. 4.

\section{B. La cooperación como estrategia para fomentar la confianza entre los Estados}

\section{Al referirnos a la confianza, ¿de qué hablamos?}

En las relaciones internacionales en general y en las que naturalmente surgen entre los Estados en el ejercicio de la actividad que se vive en el espacio ultraterrestre, la confianza es un elemento vital. Esa confianza, como símbolo de seguridad, es la que garantiza, en últimas, un clima de paz, transparencia y estabilidad.

Es importante que el término sea entendido más allá de su uso cotidiano, entendiéndolo como un "componente producto de la previsibilidad y credibilidad" (Palma, 1999, párr. 3) entre Ios Estados y como una herramienta para reducir el temor: Tal y como lo diría Luhmann desde su perspectiva sociológica: "La confianza constituye una forma más efectiva de reducción de la complejidad." (Luhmann ctd. En: Niklas, 1973, p. 16). 
Como en cualquier relación humana, la confianza entre Estados debe cultivarse, por lo que resulta indispensable que exista voluntad de parte de los Estados involucrados. La confianza va a crecer a medida que la conducta de aquellos sea la de demostrar su disposición para obrar y comportarse de forma colaborativa (Asamblea General de las Naciones Unidas, 1994, Estudio sobre la aplicación de medidas de fomento de la confianza en el espacio ultraterrestre, p. 30) $y$, en el evento en que sea posible, eliminar el mayor número de malentendidos. Los Estados deben tratar de seguir objetivos comunes y encontrar caminos similares de alcanzarlos.

\section{La confianza como elemento esencial en la exploración y utilización del espacio ultraterrestre}

Hay ciertas características propias del espacio ultraterrestre que hacen que la confianza pase a ocupar un lugar protagónico. La alta capacidad de intrusión (sobre todo de las grandes potencias) y el hecho que las naciones y la comunidad internacional, en general, dependan cada vez más de la tecnología espacial para lograr objetivos económicos y sociales en la tierra, son circunstancias que reflejan la necesidad de evitar riesgos.

En relación al nivel de intrusión, podemos decir que empezó en 1957 cuando la ex Unión Soviética lanzó el primer satélite Sputnik al espacio ultraterrestre. Más adelante, Estados Unidos ocupó su lugar con su alta capacidad militar y sus avances a nivel tecnológico. Desde entonces, los demás países han intentado "limitar sus acciones por el temor a las represalias que otra nación pudiera implementar, represalias que podrían contemplar una respuesta con armas similares a las usadas por el Estado agresor o incluso más mortíferas." (Havercroft, 2012, ¿Un tabú en las armas espaciales? Un análisis genealógico de la no-proliferación de armas en el espacio, p. 9)

Como consecuencia de ese alto grado de injerencia, hoy en día son claras muchas de las preocupaciones que aterran tanto a los países desarrollados como a los que están en vía de desarrollo. Según lo establecido por Naciones Unidas, se presentan las siguientes:

- Armamentización del Espacio Ultraterrestre;

- Utilización de sistemas espaciales militares en apoyo de operaciones de combate terrestre (satélites militares);

- Proliferación de la tecnología de los misiles en el mundo. (Asamblea General de las Naciones Unidas, 1994, p. 45).

Como segunda característica, es importante mencionar que para la vida en la tierra ha sido y sigue siendo fundamental el progreso que ha tenido la tecnología en el espacio. A nivel de las comunicaciones, por ejemplo, los satélites han jugado un papel sumamente importante.

En la década de 1960, compañías como AT\&Ty RCA se asociaron con la NASA y otras agencias espaciales para construir satélites y colocarlos en la órbita terrestre. Los sistemas de posicionamiento global, las cadenas de televisión, las estaciones de radio y los proveedores de tele- 
fonía celular dependen de estos dispositivos. (Adamu, 2012, p. 20).

Para citar otro ejemplo, se presenta el caso de la observación de la tierra. En la actualidad "es posible obtener desde el espacio ultraterrestre imágenes de cada centímetro cuadrado de la tierra con sorprendente precisión y, sobre todo, con mucha mayor nitidez" (Williams, 2008, párr. 6). Esto le permite a los países estar al tanto de posibles cambios ambientales, como por ejemplo, alteraciones climáticas o la pérdida de recursos.

En este orden de ideas, todo el panorama espacial "permite el funcionamiento de sistemas con potencial de crecimiento en ámbitos como las telecomunicaciones, la navegación y la observación de la Tierra" (Comisión Europea, 2013, Comunicación de la Comisión al Parlamento Europeo, al Consejo, al Comité Económico y Social Europeo y al Comité de las Regiones. Políticas de la UE sobre industria espacial. Aprovechar el potencial de crecimiento económico en el sector espacial, p. 3), garantizándole a los países cierto grado de independencia, innovación y competitividad, pero evidenciando también la importancia de forjar una confianza entre ellos.

\section{El consenso como generador de confianza}

La confianza entre los Estados, en el marco de la actividad espacial, debe empezar entre ellos desde el momento mismo en que se aceptan las reglas mínimas de comportamiento, es decir, desde el instante en el que se toma la de- cisión de funcionar bajo unos principios y de conformidad con el espíritu de un determinado sistema.

Las actividades de utilización y exploración del Espacio Ultraterrestre siempre han estado bajo la supervisión de Naciones Unidas, por lo tanto, el Tratado de 1967 y los demás acuerdos y convenios que constituyen el Corpus luris del espacio fueron elaborados con base en los lineamientos y principios propuestos en la Carta de la ONU.

En este sentido, al ser parte de Naciones Unidas y estar cobijados por la Resolución 2625 (XXV) de la Asamblea General del 24 de octubre de 1970, los Estados están comprometidos a guiar sus acciones hacia "el mantenimiento de la paz y la seguridad internacional y el fomento de las relaciones de amistad y de la cooperación entre las naciones" (Asamblea General de las Naciones Unidas, 1970, Art. 1) y a orientarse bajo los siguientes principios:

Los Estados, en sus relaciones internacionales, se abstendrán de recurrir a la amenaza o al uso de la fuerza contra la integridad territorial o la independencia política de cualquier Estado, o en cualquier otra forma incompatible con los propósitos de las Naciones Unidas;

Los Estados arreglarán sus controversias internacionales por medios pacíficos de tal manera que no se pongan en peligro ni la paz y la seguridad internacional ni la justicia;

La obligación de los Estados de cooperar entre sí, de conformidad con la Carta; 
Los Estados cumplirán de buena fe las obligaciones contraídas por ellos de conformidad con la Carta. (Asamblea General de las Naciones Unidas, 1970, Art. 1).

Así las cosas, independientemente que los Estados hayan o no ratificado el Tratado del Espacio y los demás instrumentos que regulan la actividad espacial, como es el caso de la resoluciones de Naciones Unidas que no tienen carácter vinculante, debe existir entre ellos un sentimiento de confianza recíproca que permita que un Estado determinado tenga la plena seguridad que otro Estado cualquiera va a guiar sus acciones sobre la base de la cooperación por el solo hecho de hacer parte del sistema de Naciones Unidas y haber consensuado en ello, es decir, haber dado su palabra.

Ese consenso, en el marco de las relaciones internacionales, ha sido reconocido como la herramienta capaz de crear un orden mundial más estable y duradero, garantizando la paz. (Hobbes ctd. En Tickner , 1997, párr. 27).

Cuando los Estados toman decisiones por consenso, implícitamente están reconociendo la necesidad de llegar a un acuerdo respecto de un asunto en específico, es decir, surge entre ellos una conciencia colectiva de luchar por una meta común y, para ello, unificar principios. En este sentido, el método del consenso "va más allá del concepto de mayoría. Reemplaza el liderazgo tradicional por el poder y la responsabilidad compartidos por todos los representantes del grupo" (Briggs, 1998, p.1). A través del él, "un grupo puede transformarse en una verda- dera comunidad y una fuerza para la trasformación social." (Briggs, 1998, p.1).

De esta manera, si a partir de un diálogo libre (aun si esta permeado por intereses) los Estados llegan a un consenso, se genera en toda la comunidad de Estados intervinientes una confianza en que todos actuarán según lo que han acordado y a lo que se han comprometido.

\section{4. ¿Cómo lograr esa confianza?}

Esa confianza de la que hemos venido hablando puede lograrse a través de lo que se conoce, a nivel internacional, como Medidas de Fomento de Confianza (MFC). Éstas tienen como propósito:

prevenir situaciones de crisis y de conflicto, tratando de fortalecer la paz y la seguridad internacional, contribuyendo al desarrollo de un mejor entendimiento, de relaciones más estables entre las naciones, creando y perfeccionando las condiciones necesarias para una cooperación provechosa. (Junta Interamericana de Defensa, 1995, Consideraciones generales sobre las medidas de fomento de la confianza mutua de carácter militar, p. 1).

Los Estados deben adoptar estas medidas de acuerdo a sus condiciones políticas, económicas, culturales y de desarrollo. Sin embargo, aún teniendo en cuenta las capacidades de cada uno de los Estados, en el espacio ultraterrestre es indispensable que éstos asuman las siguientes MFC: 


\subsection{Un constante intercambio de información}

Entre los Estados debe existir una dinámica continua de intercambio de información sobre aqueIlos aspectos que resulten ser de interés general, como aquellos relacionados a la política espacial, los verdaderos objetivos de las actividades a desarrollar, las iniciativas de nuevos proyectos, las opiniones sobre nuevos adelantos científicos y tecnológicos, las capacidades de los países en desarrollo, la experiencia de las grandes potencias, las posiciones orbitales, entre otros.

\subsection{Un sistema de notificaciones previas}

También debe existir entre los Estados un ejercicio de notificación previa, sobre todo cuando se trate de avisar acerca de lanzamientos de vehículos espaciales, objetos lanzados al Espacio UItraterrestre y maniobras y movimientos militares.

\subsection{Una evaluación y modificación del actual régimen jurídico del espacio ultraterrestre}

Es una realidad que las disposiciones normativas que regulan actualmente la actividad espacial, no son suficientes. Para citar un ejemplo, tal y como lo han sostenido países como Méxi$\mathrm{co}^{1}$ y Cuba ${ }^{2}$, el régimen jurídico actual no tiene

1 En la Tercera Conferencia de las Naciones Unidas sobre la Exploración y Utilización del Espacio Ultraterrestre con Fines Pacíficos, la delegación de México hizo referencia a la necesidad de revisar la situación actual de los tratados, acuerdos y convenios que constituyen el régimen jurídico del espacio ultraterrestre y a la importancia de fortalecer dicho régimen en lo relacionado a la "no militarización." (1998, párr. 12).

2 La intervención de la delegación de Cuba en el debate temático sobre el espacio ultraterrestre (aspectos de desarme), llevada a cabo el poder de impedir una carrera de armamentos en el espacio ultraterrestre, de allí resulta vital adecuar el Tratado de 1967 y los demás acuerdos y convenios que se derivan de él a lo planteado por Naciones Unidas en su Resolución A/53/583 sobre "Prevención de la carrera de armamentos en el espacio ultraterrestre". En esta Resolución, Naciones Unidas, reconociendo el interés de toda la humanidad en la utilización y exploración del Espacio Ultraterrestre y la importancia de llevar a cabo estas actividades con fines pacíficos, afirmó la necesidad de contar con la disposición de los Estados en la prevención de la carrera de armamentos y resaltó el papel protagónico, que en este sentido, debe tener la Conferencia de Desarme "en su calidad de único foro multilateral de negociaciones" (1998, Art. 5.

\section{La cooperación internacional como norma de lus Cogens}

Teniendo en cuenta todo lo anterior y, haciendo especial énfasis en que el régimen jurídico que vela por el orden y el uso pacífico del Espacio UItraterrestre se desprende de la reglamentación que ha hecho la Organización de las Naciones Unidas (ONU) al respecto y, en especial, aquella resumida en la Resolución 2625 de la Asamblea General, puede decirse que la cooperación internacional es una norma de lus Cogens y que así debe ser reconocida por los Estados.

el 19 de octubre de 2009, puede resumirse en los siguientes puntos: la necesidad de contribuir al uso pacífico del espacio ultraterrestre y la prevención del emplazamiento de armas. 
El hecho que la cooperación sea entendida como tal, le trae ciertas ventajas en relación a otros principios. Según lo consagrado en el artículo 53 de la Convención de Viena sobre el Derecho de los Tratados, una norma de lus Cogens es "aquella que es aceptada y reconocida por la comunidad internacional, que no admite acuerdo en contrario y que sólo puede ser modificada o derogada por una norma que presente el mismo carácter."(1969). Asimismo, se caracteriza por incorporar valores que han sido reconocidos a nivel internacional como fundamentales y por tener el poder de limitar la autonomía de la voluntad de los Estados.

En este orden de ideas, la cooperación internacional está en la cúspide de las fuentes del derecho que regulan la actividad en el espacio ultraterrestre y por lo tanto debe ser un elemento esencial en cada una de las políticas internas de los diferentes Estados.

\section{CONCLUSIÓN}

Cuando se afirma que las resoluciones, convenios y tratados que componen el marco normativo del espacio ultraterrestre se firmaron bajo la filosofía del consenso de la comunidad internacional, no se desprende ningún tipo de discusión. Es evidente que para llegar a este tipo de acuerdos, los Estados involucrados se vieron en la tarea de ceder en sus posiciones y darle prioridad a ese objetivo común que todos buscaban y que en el escenario de la actividad espacial hace referencia al uso pacífico del espacio ultraterrestre y a la actividad en beneficio de la humanidad entera.
Sin embargo, esos acuerdos (aún vigentes) solo garantizaron una completa seguridad del espacio en esa época. En su momento, cuando tuvo lugar la guerra fría, sirvieron para "prohibir el emplazamiento de armas de destrucción en masa en el espacio y prohibir la utilización militar de la luna y otros cuerpos celestes" (Asamblea General de las Naciones Unidas, 2009, Medidas para fomentar la transparencia y la confianza y propuestas sobre un tratado para la seguridad en el espacio, p. 1). Pero hoy en día, han aparecido otros problemas en el espacio que no pueden solucionarse únicamente aplicando el Tratado de 1967 o los demás convenios derivados de él. En este sentido, las controversias que han surgido en relación a temas como: la presencia militar en el espacio, "la chatarra espacial que resulta de los ensayos de armas antisatélites [...] o incluso las colisiones entre objetos que ya hay en el espacio podrían comenzar a limitar la utilización sostenible y en condiciones de seguridad del espacio ultraterrestre con fines pacíficos" (2009, p. 2) y, de esta manera, evidenciar la urgencia de nuevos consensos.

Así las cosas, se necesitan nuevas normas de comportamiento en el espacio ultraterrestre que sean consecuentes con los nuevos avances científicos y tecnológicos y con todas aquellas actividades que ahora parecen sobresalir en ese campo. Y como se explicó en un inicio, para que esa nueva reglamentación sea posible y la indicada para llenar todos esos vacíos, debe haber cooperación, consenso y confianza entre los Estados que tienen, y aquellos que luchan por tener, un lugar en la actividad espacial. 


\section{Bibliografía}

Adamu, Z. (2012). Cómo la exploración espacial cambió la vida en la tierra. CNN México. Recuperado de http://mexico.cnn.com/tecnologia/2012/10/28/como-la-exploracionespacial-cambio-la-vida-en-la-tierra

Asamblea General de las Naciones Unidas. (1959). Cooperación internacional en la utilización del espacio ultraterrestre con fines pacíficos. Aprobada por la Resolución 1472 (XIV). Recuperado de http://www.unoosa.org/oosa/SpaceLaw/gares/html/gares_14_1472.html

--. (1963). Declaración de los principios legales que deben regir las actividades de los Estados en la exploración y uso del espacio ultraterrestre. Aprobada por la Resolución 1962 (XVII), de 13 de diciembre de 1963. Recuperado de http://www.oosa.unvienna.org/pdf/ publications/STSPACE11S.pdf

---. (1966). Tratado sobre los Principios que deben regir las Actividades de los Estados en la Exploración del Espacio Ultraterrestre, incluida la Luna y otros Cuerpos Celestes. Aprobado por la Resolución 2222 (XVI), de 19 de diciembre de 1966. Recuperado de http:// www.oosa.unvienna.org/pdf/publications/ STSPACE11S.pdf

--. (1967). Acuerdo sobre el salvamento y la devolución de astronautas y la restitución de objetos lanzados al espacio ultraterrestre. Aprobado por la resolución 2345 (XXII), de 19 de diciembre de 1967. Recuperado de http://www.oosa.unvienna.org/pdf/publications/STSPACE11S.pdf

---. (1967). Tratado sobre los principios que deben regir las actividades de los Estados en la exploración y utilización del espacio ultraterrestre, incluso la Luna y otros cuerpos celestes. Aprobado por la resolución 2222 (XXI), de 19 de diciembre de 1966. Recuperado de http://www.oosa.unvienna.org/pdf/publications/STSPACE11S.pdf

---. (1969). Convención de Viena sobre el Derecho de los Tratados. Recuperado de http:// www.derechos.org/nizkor/ley/viena.html

---. (1970). Declaración relativa a los principios de derecho internacional referentes a las relaciones de amistad y a la cooperación entre los estados de conformidad con la Carta de las Naciones Unidas. Aprobada por la Resolución 2625 (XXV), de 24 de octubre de 1970. Recuperado de http://www.aloj.us.es/ eulalia/derecho\%20internacional/materiales\%20dpto/resolucion2625.htm

---. (1971). Convenio sobre la responsabilidad internacional por daños causados por objetos espaciales. Aprobada por la Resolución 2777 (XXVI), de 29 de noviembre de 1971. Recuperado de http://daccess-dds-ny.un.org/ doc/RESOLUTION/GEN/NRO/332/55/IMG/ NR033255.pdf?OpenElement 
---. Convenio sobre el registro de objetos lanzados al espacio ultraterrestre. Aprobado por la Resolución 3235 (XXIX), de 12 de noviembre de 1974. Recuperado de http:// www.oosa.unvienna.org/pdf/publications/ ST_SPACE_51S.pdf

---. (1994). Estudio sobre la aplicación de medidas de fomento de la confianza en el espacio ultraterrestre. New York, NY: Naciones Unidas.

---. (1997). Declaración sobre la cooperación internacional en la exploración y utilización del espacio ultraterrestre en beneficio e interés de todos los Estados, teniendo especialmente en cuenta las necesidades de los países en desarrollo. Aprobada por la Resolución $51 / 122$, de 4 de febrero de 1997. Recuperado de http://www.dipublico.com.ar/3991/ declaracion-sobre-la-cooperacion-internacional-en-la-exploracion-y-utilizacion-del-espacio-ultraterrestre-en-beneficio-e-interes-detodos-los-estados-teniendo-especialmenteen-cuenta-las-necesidades/

---. (1998). Prevención de una carrera de armamentos en el espacio ultraterrestre. Aprobada por la Resolución A/53/583, 15 de septiembre de 1998. Recuperado de http:// www.unoosa.org/pdf/gadocs/A_53_583S. pdf

---. (2009). Medidas para fomentar la transparencia y la confianza y propuestas sobre un tratado para la seguridad en el espacio. Canadá: Documento de Trabajo. Recuperado de
http://www.unog.ch/80256EDD006B8954/ (httpAssets)/619E3027A8BC100BC12575F C003BF139/\$file/CD_1865_S.pdf

Briggs, B. (1998). Introducción al proceso de consenso. Recuperado de http://caminosostenible.org/wp-content/uploads/BIBLIOTECA/introduccion-al-proceso-de-consenso--beatrice-briggs.pdf

Comisión Europea. (2013). Comunicación de la Comisión al Parlamento Europeo, al Consejo, al Comité Económico y Social Europeo y al Comité de las Regiones. Políticas de la UE sobre industria espacial. Aprovechar el potencial de crecimiento económico en el sector espacial. Recuperado de http:// eur-lex.europa.eu/LexUriServ/LexUriServ. do?uri=COM:2013:0108:FIN:ES:PDF

Consejo de Estudios Internacionales Avanzados. (1981). Mesa redonda sobre solución de controversias en derecho espacial: rounde tableo n Settlement of Space Law disputes. Córdoba: Casa de la Cultura.

Delegación de Cuba ante la Asamblea General de las Naciones Unidas. (2009). Intervención de la delegación de Cuba en el debate temático sobre el espacio ultraterrestre (aspectos de desarme). Recuperado de http://anterior. cubaminrex.cu/AGNU/Articulos/64Periodo/ Intervenciones/091019_3.html

Delegación de México ante la Asamblea General de las Naciones Unidas. (1998). Intervención de la delegación de México ante la 
Tercera conferencia de las Naciones Unidas sobre la exploración y utilización del espacio ultraterrestre con fines pacíficos. Recuperado de http://mexicoenelespacio.blogspot. com/2009/05/intervencion-de-la-delegacion-de-mexico.html

Espinosa Acuña O., y Vaca Gonzalez, P. (2012). Comercio Exterior, Cooperación Internacional y Progreso Técnico desde la Teoría del Desarrollo Económico de la CEPAL y de Raúl Prebisch. En L. Ivarola. (Comp.). Selección de Trabajos de las XVII Jornadas de Epistemología de las Ciencias Económicas. 27-36. Buenos Aires: Universidad de Buenos Aires, Facultad de Ciencias Económicas.

Grupo de políticas económicas y grupo de economía para el desarrollo, PREM. (2000). ¿Qué es la globalización? Banco Mundial. Recuperado de http://www.bancomundial.org/ temas/globalizacion/cuestiones1.htm\#top

Havercroft, J. (2012). ¿Un tabú en las armas espaciales? Un análisis genealógico de la no-proliferación de armas en el espacio. Escenarios XXI, 2(13), 5-32 Recuperado de http://www.academia.edu/1903747/_Un_ tabu_en_las_armas_espaciales_Un_analisis_genealogico_de_la_no-proliferacion_de_ armas_en_el_espacio

Junta Interamericana de Defensa. (1995). Consideraciones generales sobre las medidas de fomento de la confianza mutua de carácter militar. Recuperado de http://www.fasoc. cl/files/articulo/ART41192a8f46afc.pdf
Kopal, V. (2009). Tratado sobre los principios que deben regir las actividades de los Estados en la exploración y utilización del espacio ultraterrestre, incluso la Luna y otros cuerpos celestes. Naciones Unidas. Recuperado de http://legal.un.org/avl/pdf/ha/tos/ tos_s.pdf

Lachs, M. (1977). El derecho del espacio ultraterrestre. México D.F.: Fondo de Cultura Económica.

Mayoral, F. La globalización y el ritmo de integración de los países en desarrollo. Salamanca: Universidad de Salamanca, Facultad de Derecho. Recuperado de http://pendientedemigracion.ucm.es/info/cecal/encuentr/ areas/economia/2e/martin.htm

Niklas, L. (1973). Confianza. México: Anthopos/ UIA. Citado por: Hevia De La Jara, F. (2003). ¿Cómo construir confianza? Hacia una definición relacional de la confianza social. México: UNAM. Instituto de Investigaciones Jurídicas.

Palma, H. (1999). Desarme y seguridad: una nueva agenda de América Latina y el Caribe para el próximo milenio, Medidas Regionales de Fomento a la Confianza. Perú: Seminario Internacional sobre Desarme. Recuperado de http://www.opanal.org/Articles/SeminarLima/palma_lima_e.htm

Rodríguez Medina, E. (s.f.). Nuestro Derecho al Espacio. La órbita geoestacionaria: ¿una frustrada regulación? México DF: Biblioteca 
Jurídica Virtual del Instituto de Investigaciones de la UNAM. Recuperado de http://www. juridicas.unam.mx/publica/librev/rev/juicio/cont/2/cnt/cnt4.pdf

Secretaría Ejecutiva. (2002). Globalización y desarrollo. Naciones Unidas, CEPAL. Recuperado de http://www.eclac.org/cgi-bin/getProd. asp?xml=/publicaciones/xml/6/10026/ P10026.xmI\&xsI=/publicaciones/ficha. xsl\&base=/publicaciones/top_publicaciones.xs|
Tickner. A. (1997). Hacia un modelo normativo del consenso en las instituciones internacionales. Colombia Internacional, 38, 3-11. Recuperado de http://colombiainternacional. uniandes.edu.co/view.php/252/index=1. php?action $=$ edit\&id $=252 \# 8$

Williams, S. (2008). La información obtenida por tecnologías espaciales ante el derecho internacional. Recuperado de http://www. scielo.org.ve/scielo.php?pid=S1690515200 $8000200005 \&$ script=sci_arttext 